\title{
Risk Evaluation of Recovery Efficiency Based On Orthogonal Experiment and Monte Carlo Method
}

\author{
Zhang Yalong \\ Chongqing University of Science and Technology \\ China \\ e-mail:550732595@qq.com
}

Pang Jin

Chongqing University of Science and Technology China

Crab1981@126.com

\author{
Liu Hong \\ Chongqing University of Science and Technology \\ China \\ e-mail:liubrett@vip.sina.com \\ Gao Dewei \\ Southwest Oil and Gas Field Branch, Petrochina \\ China \\ e-mail:Gao.dw777@gmail.com
}

\begin{abstract}
The parameters (such as the permeability and the porosity, etc) that affect the recovery efficiency of oil reservoir have the uncertainties within a certain range, which will bring certain risks to the evaluation on recovery efficiency of the numerical simulation program. According to the analysis of the orthogonal experiment and the Monte Carlo method, the evaluation method of recovery efficiency based on the link application of the above two methods has been proposed in this paper. Firstly, the orthogonal table has been created through defining the uncertain parameters and their ranges. Afterwards, the multiple linear relationship between the uncertain parameters and the recovery efficiency has been established according to the numerical simulation of different experiment programs. Finally, the Monte Carlo method has been adopted in this paper to simulate the distribution frequency of recovery efficiency when the uncertain parameter shows a normal distribution, which will provide the reliable basis for the determination of recovery efficiency.
\end{abstract}

Keywords-orthogonal experiment; Monte Carlo; recovery efficiency; risk; program

\section{INTRODUCTION}

With the increase of the difficulty of oil and gas resources development, it is imperative to increase the oil recovery.The study of influence factor about oil recovery is vital for oil field development[1,2]. Reservoir system is a complex nonlinear system,therefore, in order to solve the statistical simulation problem of oil recovery,we first put forward the optimum structure of model and then build reliable statistical model according short source.Now, the method of calculating the recovery are formula method, BP model method,polymer flooding model[3,4,5],but these method can not be analyzed intuitive.This paper introduce the method of orthogonal experiment and monte carlo to analyze the parameters of oil recovery intuitively and then get the pecking order of different parameters so that it can provide basis for EOR.

\section{PRINCIPLE OF ORTHOGONAL EXPERIMENT}

The orthogonal experiment design is a design method to study various factors and various levels, which will select some representative points from the full-scaling experiment for the test according to the orthogonality. These representative points have the characteristics of "homodisperse and comparable uniformity". Therefore, it is considered as a highly-efficient, rapid and economic experimental design method[6,7].

The experiment procedures can be shown as follows:

(1) Determining the number of lists: according to the purpose of experiment, it is required to select the processing factors and the non-ignorable interaction. At the same time, the number should be clearly determined. If some issues in this study cannot be understood, the lists can be more. But the number should be appropriate.

(2) Determining the levels of factors: according to the research purpose, the general two levels (yes or no) can be used in the factor screening.

(3) Selecting the orthogonal table: it is required to select the appropriate orthogonal table according to the determined lists and levels.

(4) Arranging the head of table: it is required to prior consider the non-ignorable processing factors of interactions. According to the non-mixed principle, these factors and the interactions should be properly arranged in the head of table. Afterwards, the remaining factors should be randomly arranged in each list.

(5) Organizing the implementation program: it is required to constitute the implementation program table according to the level series of the lists of various factors. It should be conducted according to the experimental number and the experiment should be repeated for $n$ times. At the same time, each experiment should be performed by the rampant level combination in the table.

\section{PRINCIPLE OF MONTE CARLO METHOD}

The Monte Carlo method refers to the numerical method which adopts any sequence of random numbers to 
conduct the stochastic simulation[8,9]. It is also known as the random sampling technique method or the statistical test method. In the risk assessment of project, it is considered as a relatively accurate method[10,12]. The basic principle is: according to the definition of probability, the probability of an event can be estimated by the occurrence frequency of this event in a large number of trials; when the size of samples is large enough, it can be considered that the occurrence frequency of this event is its probability. Therefore, a large number of random samplings can be conducted for the random variables which will affect the reliability. Afterwards, these sample values should be substituted to the performance functions in groups so as to determine the various function values (such as the recovery efficiency). Then, the distribution frequency of various function values can be obtained.

Assuming that the random variables with statistical independence is $\mathrm{Xi}(\mathrm{i}=1,2,3, \cdots, \mathrm{k})$, the corresponding probability density functions are respectively $\mathrm{fx} 1, \mathrm{fx} 2, \cdots, \mathrm{fxk}$ and the performance function is $\mathrm{Z}=\mathrm{g}(\mathrm{x} 1, \mathrm{x} 2, \cdots, \mathrm{xk})$. Firstly, according to the corresponding distribution of various random variables, it is required to generate $\mathrm{N}$ groups of random number $\mathrm{x} 1$, $\mathrm{x} 2, \cdots, \mathrm{xk}$. At the same time, it is also necessary to calculate the performance function value $\mathrm{Zi}=\mathrm{g}(\mathrm{x} 1$, $\mathrm{x} 2, \cdots, \mathrm{xk})(\mathrm{i}=1,2, \cdots, \mathrm{N})$. If the corresponding performance function value of $\mathrm{L}$ groups of random number $\mathrm{Zi} \leqslant 0$, the probability of function value as well as the reliable indicator can be obtained according to the Bernoulli law of large numbers and the characteristics of normal random variables when $\mathrm{N} \rightarrow \infty$.

\section{RISK ASSESSMENT METHOD OF RECOVERY EFFICIENCY}

There is the complex nonlinear relationship between the recovery efficiency calculated by the numerical simulation of oil reservoir and the basic data of oil reservoir adopted by the program (such as the permeability, the porosity, the bound water saturation, etc). Meanwhile, the various parameters that affect the recovery efficiency of crude oil have the certain volatility or the uncertainties. In the risk assessment of recovery efficiency proposed in this paper, the orthogonal experimental analysis method is firstly adopted to design the program. The experimental matrix should cover the interference of all parameters as much as possible. Then, the operation numbers of program simulation can be reduced and the reliability of regression can be ensured. At the same time, the response surface can be also generated. Based on the Monte Carlo principle, the random simulation calculation can be conducted through determining the random distribution of the various factors which affect the recovery efficiency of crude oil. Afterwards, the probability distribution of the recovery efficiency can be obtained so as to perform the risk assessment of recovery efficiency. The steps of specific assessment can be shown as follows:

(1) The uncertain parameters and their ranges should be defined according to the reservoir properties.

(2) It is required to adopt the experimental analysis method to establish the orthogonal table which contains all the uncertain parameters.
(3) It is necessary to define the corresponding numerical simulation program of the operation of parameters based on the orthogonal table.

(4) The linear relationship between the recovery efficiency and the uncertain parameters should be established according to the multiple linear regression.

(5) It is required to evaluate the influence of uncertain parameters on the recovery efficiency.

(6) The Monte Carlo method should be adopted to establish the distribution curve of recovery efficiency and it should be evaluated.

\section{APPLICATION}

\section{A. Determination of uncertain parameters}

According to the reservoir characteristics of a fault block, the uncertain parameters in the development process should contain the porosity $\Phi$, the bound water saturation Swi, the oil-water interface WOC, the level permeability $\mathrm{Kh}$, the residual oil saturation Sor and the vertical permeability/level permeability $\mathrm{Kv} / \mathrm{Kh}$. All the uncertain parameters are taken as the independent variables to be evaluated. The main evaluation parameters and the ranges can be shown in Table 1 .

TABLEI UNCERTAIN PARAMETERS AND THE RANGES

\begin{tabular}{|c|l|c|c|c|}
\hline \multirow{2}{*}{ Variables } & \multicolumn{1}{|c|}{ Parameters } & \multicolumn{3}{|c|}{ Ranges } \\
\cline { 3 - 5 } & Porosity, $\Phi$ & Low & Moderate & High \\
\hline $\mathrm{X}_{1}$ & Bound water saturation, $\mathrm{S}_{\mathrm{wi}}$ & $27 \%$ & $37 \%$ & $45 \%$ \\
\hline $\mathrm{X}_{2}$ & Oil-water interface, WOC & $2290 \mathrm{~m}$ & $2300 \mathrm{~m}$ & $\begin{array}{c}2310 \\
\mathrm{~m}\end{array}$ \\
\hline $\mathrm{X}_{3}$ & Level permeability, $\mathrm{K}_{\mathrm{h}}$ & $0.5 \mathrm{~K}_{\mathrm{h}}$ & $\mathrm{K}_{\mathrm{h}}$ & $2 \mathrm{~K}_{\mathrm{h}}$ \\
\hline $\mathrm{X}_{4}$ & Residual oil saturation, $\mathrm{S}_{\mathrm{or}}$ & $25 \%$ & $31 \%$ & $35 \%$ \\
\hline $\mathrm{X}_{5}$ & $\begin{array}{l}\text { Vertical permeability/Level } \\
\text { permeability, } \mathrm{K}_{\mathrm{v}} / \mathrm{K}_{\mathrm{h}}\end{array}$ & 0.3 & 0.65 & 1 \\
\hline $\mathrm{X}_{6}$ & & & & \\
\hline
\end{tabular}

\section{B. Regression analysis}

According to the orthogonal experimental analysis, nine groups of data combination have been designed for the uncertain parameters of this fault block. Afterwards, the corresponding numerical simulation has been conducted and the recovery efficiency has been considered as the research object. The simulation results can be shown in Table 2. " 0 ", " -1 " and " +1 " in the experimental matrix respectively represent the moderate situation, the minimum situation and the maximum situation. This matrix contains all the possible highest and lowest combinations. 
TABLEII

DESIGN MATRIX OF PROGRAM

\begin{tabular}{|c|c|c|c|c|c|c|c|}
\hline Program & $\Phi$ & $\mathrm{S}_{\mathrm{wi}}$ & WOC & $\mathrm{K}_{\mathrm{h}}$ & $\mathrm{S}_{\mathrm{or}}$ & $\mathrm{K}_{\mathrm{v}} / \mathrm{K}_{\mathrm{h}}$ & $\begin{array}{c}\text { Recovery } \\
\text { efficiency } \\
(\%)\end{array}$ \\
\hline 1 & 1 & -1 & -1 & 1 & -1 & 1 & 18.9 \\
\hline 2 & 1 & 1 & -1 & -1 & 1 & -1 & 24.3 \\
\hline 3 & 1 & 1 & 1 & -1 & -1 & 1 & 34.7 \\
\hline 4 & -1 & 1 & 1 & 1 & -1 & -1 & 32.5 \\
\hline 5 & 1 & -1 & 1 & 1 & 1 & -1 & 31.9 \\
\hline 6 & -1 & 1 & -1 & 1 & 1 & 1 & 20.4 \\
\hline 7 & -1 & -1 & 1 & -1 & 1 & 1 & 29.2 \\
\hline 8 & -1 & -1 & -1 & -1 & -1 & -1 & 18.6 \\
\hline 9 & 0 & 0 & 0 & 0 & 0 & 0 & 21.4 \\
\hline
\end{tabular}

It is required to establish the multiple linear regression equation according to the numerical simulation results. The relational equation between the recovery efficiency and the uncertain parameters can be shown as

$\mathrm{Y}=-13.0065+0.1121 \mathrm{X} 1-0.1902 \mathrm{X} 2+0.0058 \mathrm{X} 3$ $0.0036 \times 4-0.0430 \times 5-0.02670 \times 6$.

The relationship between the recovery efficiency predicted by the regression equation and the recovery efficiency of numerical simulation can be shown in Fig .1. There is a linear relationship between them and the correlation coefficient is 0.9545 . It is indicated that the response surface of this equation can better conclude the general trend of simulation model and it has the good representativeness.

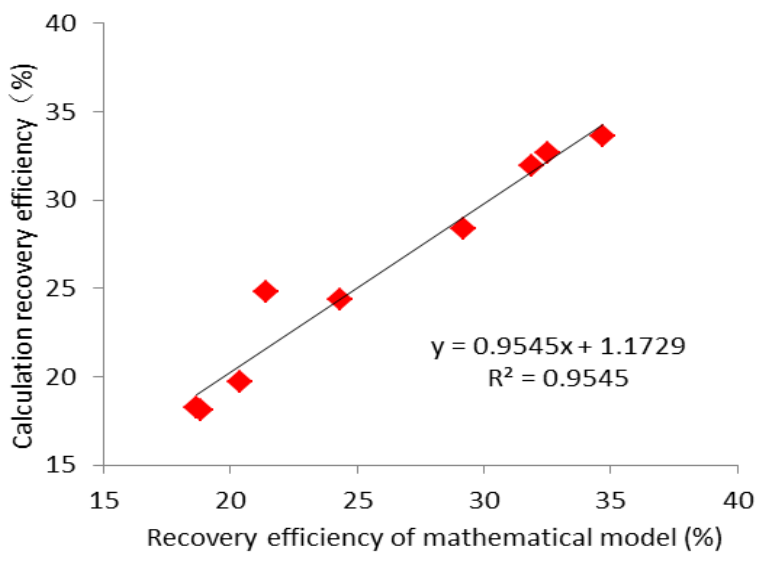

Figure 1. The recovery efficiency predicted by the regression equation and the recovery efficiency of numerical simulation.

According to the response situation of different programs, it is required to adopt the least square method to analyze the influence of each uncertain parameter on the response variables (recovery efficiency). According to the significant analysis of the normal distribution of uncertain parameters (Fig .2, the confidence level is 95\%), the influence of Sor and $\mathrm{Kh}$ on the recovery efficiency is the maximum, followed by $\mathrm{Kv} / \mathrm{Kh}$, Swi and $\Phi$. The influence of WOC on the recovery efficiency is the minimum.

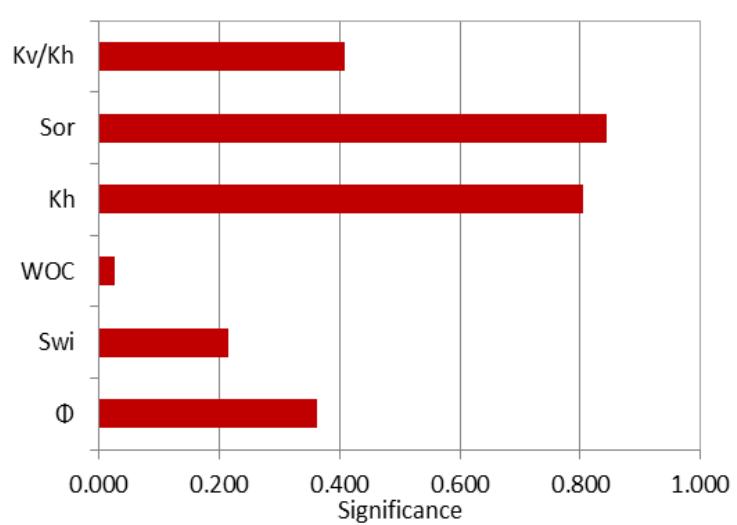

Figure 2. Analysis on the influence of uncertain parameters

\section{Analysis on the distribution of recovery efficiency}

According to the Monte Carlo principle, it is required to adopt the regressive multiple linear equation to predict the recovery efficiency of this fault block. The six uncertain parameters should be valued in normal distribution and the mid-value is the intermediate value. The Monte Carlo simulation is performed for 1000 times.

The histograms of recovery efficiency and the normal distribution function can be shown in Fig .3 and the cumulative distribution curve of recovery efficiency can be shown in Fig .4. It is found that the recovery efficiency of P10, P50 and P90 is respectively $30 \%, 26 \%$ and $21 \%$. In other words, the probability that the recovery efficiency is up to $21 \%$ is above $90 \%$ and the probability that the recovery efficiency is up to $30 \%$ is less than $10 \%$ when the nature of this fault block is not completely determined.

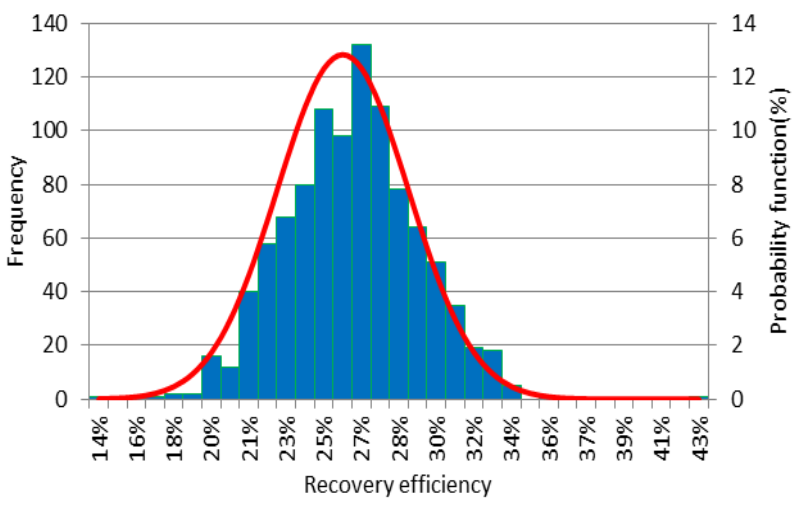

Figure 3. The histogram of the distribution of recovery efficiency 


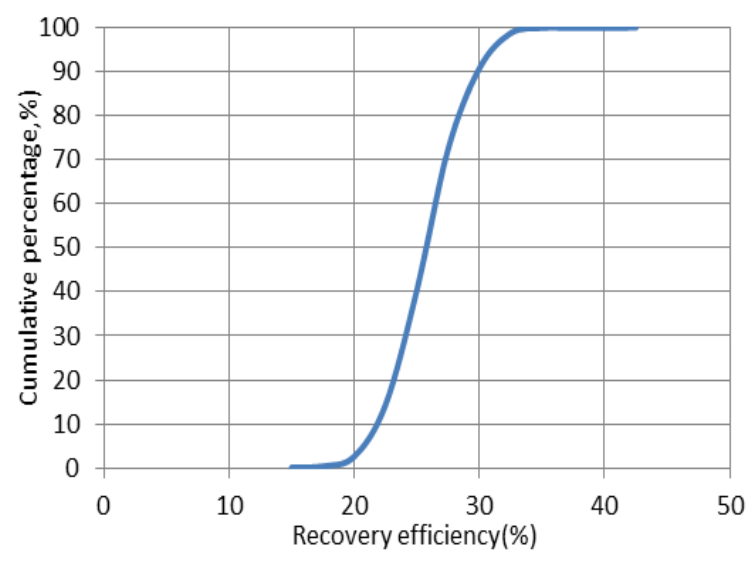

Figure 4. The cumulative distribution curve of recovery efficiency

\section{CONCLUSION}

(1) The orthogonal experiment method arranges the experiment according to the table in the process of evaluating the recovery efficiency. It is convenient, the points are distributed in balance and the number of trials is fewer. At the same time, it has the orthogonality, which can easily analyze the dominant factors that affect the changes of recovery efficiency.

(2) The random distribution of the uncertain factors that affect the recovery efficiency has been considered in Monte Carlo method. A more accurate probability distribution of recovery efficiency can be obtained if the number of simulation is sufficient.

(3)As the reservoir parameters have the uncertain risk assessment of recovery efficiency, it is required to adopt the orthogonal experiment and the Monte Carlo method to discuss the frequency distribution of recovery efficiency. The idea of this method is simple and the accuracy of calculation is high. At the same time, the number of numerical simulation has been also greatly reduced.

\section{ACKNOWLEDGMENT}

We thank the Department of Petroleum Engineering at Chongqing University of Science and Technology for providing resources and computing facilities.We gratefully acknowledge Dr. LiuH and Dr. PangJ. for their contributions during this research.

\section{REFERENCES}

[1] J bingyu, "Progress and prospects of enhanced oil recovery technologies at home and abroad".Oil \& Gas Geology, 2012,01:111-117.

[2] Q Ligang, "The influence factor and countermeasure analysis of oilfield recovery". Chemical Engineering \& Equipment, 2014,05:107-108.

[3] C Yudong and Y Linsheng." Oil recovery ratio modeling by neural network technique”.OGP,1994,29(2):166 169.

[4] C Yuanqian."A method of prediction of recovery factors in oilfields at high-water-cut stage".Xinjiang Petroleum Geology. 1994,03:247-252.

[5] W Yuming, Wang Jiaying and K Qinghong."Estabilishment and application of a prediction model for enhanced oil recovery in polymer flooding”. Acta Petrolei Sinica. 2013,03:513-517.

[6] C. Kui, Experimental Design and Analysis. Beijing: Tsinghua University Press, (2009).

[7] Z. Pingwen and L. Tiejun, Numerical Analysis. Beijing: Peking University Press, (2007).

[8] X. Shutie., Mathematical Experiment. Beijing: Higher Education Press, (2001).

[9] L. Weixuan, Experimental Design Method. Dalian: Dalian Maritime University Press, (1995)

[10] X. Zhongji, Monte Carlo Method. Shanghai: Shanghai Science and Technology Press, (1985).

[11] X. Zhongru, Regression Analysis and Experimental Design. Beijing: China Agricultural Press, (1998). 